\title{
DEVELOPMENT OF NEW EMISSION SYSTEMS OF ELECTRON BEAM GUNS FOR PROCESS OPERATIONS UNDER SPACE CONDITIONS*
}

\author{
Yu.V. ZUBCHENKO and E.G. TERNOVOJ \\ E.O. Paton Electric Welding Institute, NASU \\ 11 Bozhenko Str., 03680, Kiev, Ukraine. E-mail: office@paton.kiev.ua
}

\begin{abstract}
Development of a new system, in contrast to diode emission systems with filamentary cathode being earlier applied in space guns, is based on triode emission system with cathode preheating by electron bombardment. New emission system is designed using a procedure based on simultaneous solution of problems of analysis and synthesis applying an apparatus for trajectory analysis of electron beams. The proposed emission system is designed in two variants, i.e. manual and automated types of electron beam tools. An electron beam with phase characteristic close to linear one is formed in each of calculated variants, that provides for high specific power of the beam in place of welding (to $9 \mathrm{~kW} / \mathrm{mm}^{2}$ ) at total beam capacity to $2.5 \mathrm{~kW}$ and, as a result, allows carrying out welding and cutting of aluminum and titanium alloys, as well as stainless steels, of up to $5 \mathrm{~mm}$ thickness in continuous and pulsed modes under open space conditions. 10 Ref., 1 Table, 7 Figures.
\end{abstract}

$\boldsymbol{K} \boldsymbol{e} \boldsymbol{y} \boldsymbol{w} \boldsymbol{o} \boldsymbol{r} \boldsymbol{d} \boldsymbol{s}:$ emission system, electron beam, triode system, crossover, focal plane, trajectory analysis, perveance, operating distance, manual and automated tool, welding, cutting, brazing, heating, aluminum and titanium alloys, stainless steels

At present time the E.O. Paton Electric Welding Institute carries out works on development of a new generation EB tool for performance of erection and repair works in open space [1]. The developed tool provides for two design variants, namely manual and automated one being part of a robot or precision manipulator.

Task of the present work lies in development of emission systems of EB gun for two variants of the developed tool, which will provide increase of general (to $2.5 \mathrm{~kW}$ ) and specific (to $9 \mathrm{~kW} / \mathrm{mm}^{2}$ ) beam power in continuous pulsed mode as well as rise of service reliability of the equipment.

In contrast to EB guns with diode emission systems and filamentary cathodes [2-5] being earlier applied for work under space conditions, current problem statement requires application of triode emission system. In it the cathode is heated up by electron bombardment and operates in a mode of emission current limitation by electron beam space charge, and beam power is varied by supply of cathode, negative controlled potential, to modulated (focusing) electrode at fixed accelerating voltage and constant cathode temperature.

Let us consider general and distinctive features of beam formation in guns for manual and automated variants.

Maximum attainable current density in a crossover (minimum section of beam formed only by emission system, i.e. electrostatic part of electron-optical system of the gun) is only caused by heat velocities of the electrons and evaluated by Langmuir dependence, which for paraxial beams is reduced to [6]

$$
J_{\mathrm{CR}}=j_{\mathrm{C}} \frac{e U_{\mathrm{acc}}}{k T_{\mathrm{C}}} \gamma_{1}^{2},
$$

where $j_{\mathrm{C}}$ is the density of current emission, A $/ \mathrm{mm}^{2} ; e=1.6 \cdot 10^{-19}$ is the electron charge, C; $U_{\text {acc }}$ is the accelerating voltage, $\mathrm{V} ; k$ is the Boltzmann constant; $T_{\mathrm{C}}$ is the absolute cathode temperature, $\mathrm{K} ; \gamma_{1}$ is the beam convergence angle in the crossover, rad.

Let us analyze the ways of possible increase of current density in the crossover applicable to conditions of gun operation in space.

Reduction of cathode operating temperature $T_{\mathrm{C}}$ and increase of density of emission current from cathode $j_{\mathrm{C}}$ are virtually impossible due to limited selection of cathode materials (tantal, tungsten, lanthanum hexaboride) related with For the first time works on synthesis of emission systems of power electron beam welding guns were carried out in the PWI Department
by Drs K.S. Akopiants, Yu.V. Zunchenko and V.E. Lokshin under the leadership of Prof. O.K. Nazarenko, Corr. Member of the NASU. 
heavy operating conditions of the cathode in welding guns.

Increase of accelerating voltage more than $10 \mathrm{kV}$ is also unallowable due to danger of X-ray irradiation of operator. Rise of convergence angle $\gamma_{1}$ for increase of current density in crossover can be efficient, but practically applied only in the guns for automated variant, where final focusing of the beam on part being welded is carried out by additional electromagnetic lens transmitting crossover image in a focal plane (Figure 1, I). For manual gun variant the conditions are necessary, at which final beam focusing on the part is performed only by emission system, i.e. crossover of the beam is located in the focal plane (Figure 1, II). In this situation, increase of convergence angle is related with reduction of gun operating distance (from anode to part), that limits line-of-sight coverage of a weld pool as well as promotes increase of possibility of breakdown in welding or brazing of the materials with high vapor pressure and reduction of electric strength of gun isolating gaps.

Thus, any of the conditions, given in expression (1), cannot be used for direct increase of current density in the crossover in manual variant of the gun. In automated variant, vice versa, increase of beam convergence angle in the crossover is reasonable and efficient, since in this case the emission system shall form short-focus electron beam converging in the crossover before electromagnetic lens.

It should be noted that expression (1) is true only for idealized conditions of beam formation in the emission system (absence of aberrations, absence of influence of beam space charge, uniformity of field intensity near the cathode, constant density of emission current on cathode surface etc.). Only under these conditions, due to the fact that thermal rates of electrons are distributed by Maxwell law, distributions of current density in the crossover obey normal Gaussian law, and crossover radius acquires the minimum possible value. Therefore, measures on further improvement of structure and parameters of the emission systems in development of close to ideal conditions of the electron beam formation are efficient for rise of current density in the crossover.

Analysis of the conditions of beam formation in the emission systems was carried out using trajectory analysis apparatus from package of application programs ERA designed for automation of electron-optical calculations [7]. According to this apparatus the beam is represented in form of finite number of fine coaxial current tubes, which are displayed on longitudinal beam section
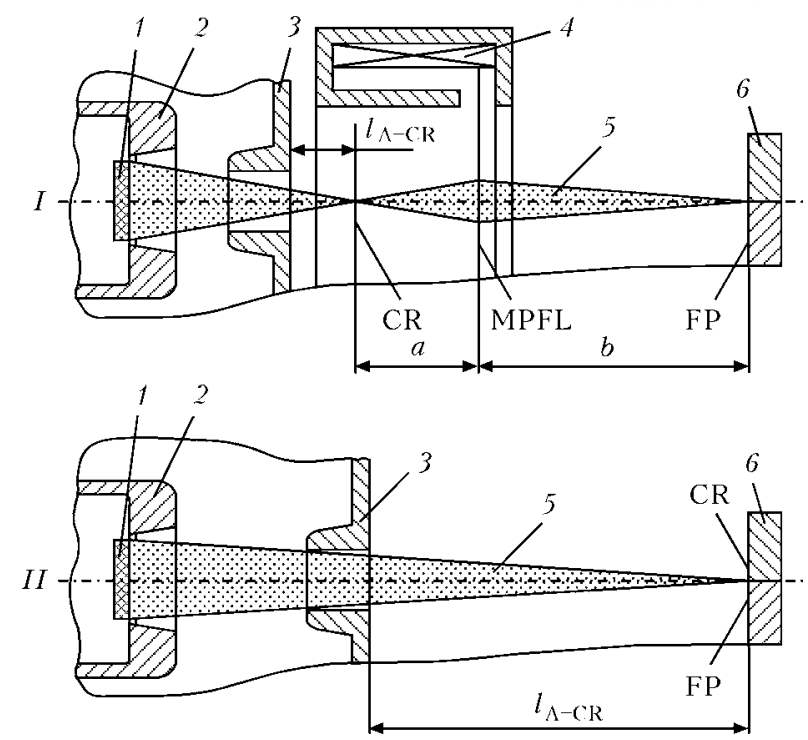

Figure 1. Schemes of emission systems of guns for EBW in space: $I$ - for automated variant; $I I$ - for manual variant; 1 - cathode; 2 - focusing electrode; 3 - anode; 4 electromagnetic focusing lens; 5 - electron beam; 6 part being welded; CR - crossover; MPFL - mean plane of focusing lens; FP - focal plane; $l_{\mathrm{A}-\mathrm{CR}}-$ anode-crossover distance; $a$ - distance along CR-MPFL system axis; $b$ - distance along MPFL-CR system axis

by corresponding number of trajectories. Spherical aberrations of analyzed emission system can be considered by means of development of phase characteristic of a beam formed in this system according to the results of trajectory analysis. Phase characteristic of the beam is represented as dependence of inclination angle $r_{i}{ }^{\prime}$ of trajectories on their radial coordinate $r_{i}$ derived for any cross-section of the beam. $r^{\prime}-r$ variables range is called phase plane [8]. If thermal rates of electrons are not considered and emission system does not have spherical aberrations, phase characteristic of the beam is linear in such a system and inclination of trajectories $r_{i}^{\prime}$ is proportional to radiuses of their location $r_{i}$, i.e.

$$
r_{1}{ }^{\prime} / r_{1}=r_{2}{ }^{\prime} / r_{2}=\ldots=r_{n}{ }^{\prime} / r_{n}=\text { const }=c,
$$

where $c$ is the inclination of phase characteristic.

Only in this ideal case all trajectories of the beam, converging after cathode, will concur in one point of the crossover at its radius $r^{*}=0$, and phase characteristic is expressed by linear dependence $r^{\prime}=c r$, where $1 / c=l_{c}$ is the distance from plane $z_{\text {ph.ch }}$, in which phase characteristic is determined, to crossover position plane $z_{\mathrm{CR}}$ (Figure 2).

In general case the following dependence is received considering $c$ sign:

$$
z_{\mathrm{CR}}=z_{\mathrm{ph} . \mathrm{ch}}-1 / c,
$$

where $c<0$ and $l_{c}=1 / c<0$ at $z_{\mathrm{ph} . \mathrm{ch}}<z_{\mathrm{CR}} ; c>$ $>0$ and $l_{c}=1 / c>0$ at $z_{\text {ph.ch }}>z_{\mathrm{CR}}$. 


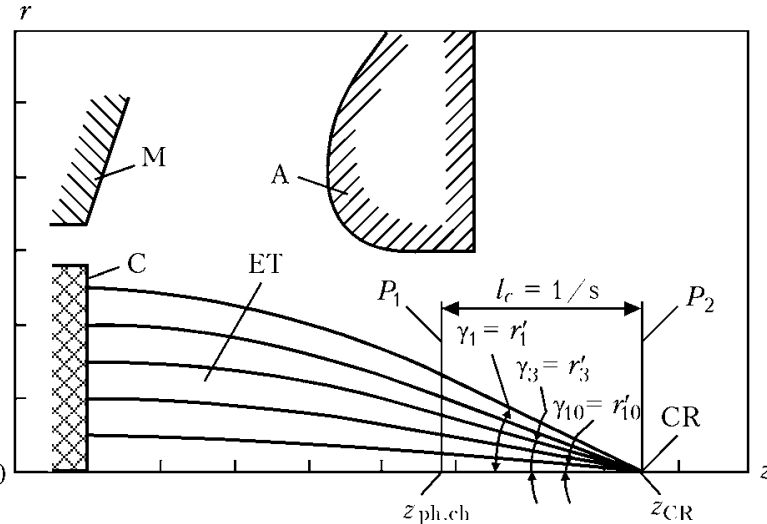

Figure 2. Image of electron trajectories of ideally formed beam: $\mathrm{C}$ - cathode; $\mathrm{M}$ - modulating (focusing) electrode; A - anode; ET - electron trajectory; $r$ - radial coordinate; $z$ - longitudinal coordinate of emission system (system axis); CR - beam crossover; $z_{\mathrm{CR}}-P_{2}$-plane $z$-coordinate (crossover position); $z_{\text {ph.ch }}-P_{1}$-plane coordinate in which beam phase characteristic is plotted; $\gamma$ - inclination angles of trajectory to $z$-axis (for paraxial beam $\gamma \approx$ $\left.\approx \operatorname{tg} \approx \gamma \approx r^{\prime}\right) ; c-$ inclination of phase characteristic of beam in $P_{1}$-plane

In general case, due to spherical aberrations of the emission systems, electron beams are nonlaminar to that or another degree, and their phase characteristics are nonlinear, respectively. Nonlinear phase characteristic in any beam section can be represented in form of

$$
r^{\prime}=c r+\varepsilon(r) .
$$

Here $c$ is the average inclination of phase characteristic; $\varepsilon(r)$ is the deviation of phase characteristic from linear one.

Analysis of phase characteristics of the beams can be significantly simplified with the help of method of phase parallelogram [9], which is plotted on phase plane $r^{\prime}-r$, in such a way as to include considered phase characteristic in its minimum area (Figure 3).

According to Liouville theory [8], phase parallelogram, plotted for any $z_{\text {ph.ch }}$ section in beyond anode area, intercept segment $r$ being equal

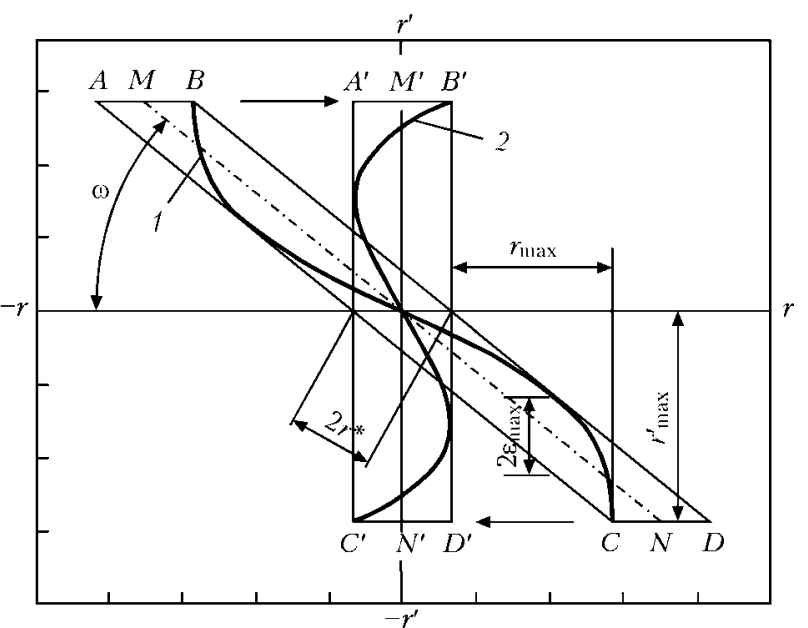

Figure 3. Plotting of phase parallelogram: 1 - phase characteristic for beam section in area before crossover; $2-$ the same in crossover (arrows show directions of parallelogram deformation in movement of investigated beam section to crossover); $A, B, C, D, M, N, A^{\prime}, B^{\prime}, C^{\prime}, D^{\prime}, M^{\prime}, N^{\prime}-$ points of parallelograms plotting; $\omega$ - inclination angle of median line of phase parallelogram to $r$-axis; $2 \varepsilon_{\max }-$ maximum deviation of phase characteristic from linear one; $2 r^{*}-$ beam diameter in crossover; $r_{\max }-$ radius of extreme trajectory; $r_{\max }^{\prime}-$ inclination angle of extreme trajectories to crossover diameter $2 r^{*}$. Finite dimensions of the crossover equal $2 r^{*}$ are caused by nonlinear phase characteristic, i.e. finite value $\varepsilon_{\max }$ of deviation of phase characteristic from linear one. Based on parallelogram plotting the following relationship can be received:

$$
r^{*}=\varepsilon_{\max } / c ; \quad c=r_{\max }^{\prime} /\left(r_{\max }+r^{*}\right) .
$$

Construction of new emission system becomes laborious and time-consuming process if only analysis method (trial-and-error) and apparatus for trajectory analysis are used. In this connection for construction of such system we used a procedure based on simultaneous solution of synthesis and analysis problems [10]. Final aim of the calculation is attaining a beam phase characteristic close to linear one, formation of electron beam with set values of its angle of convergence and

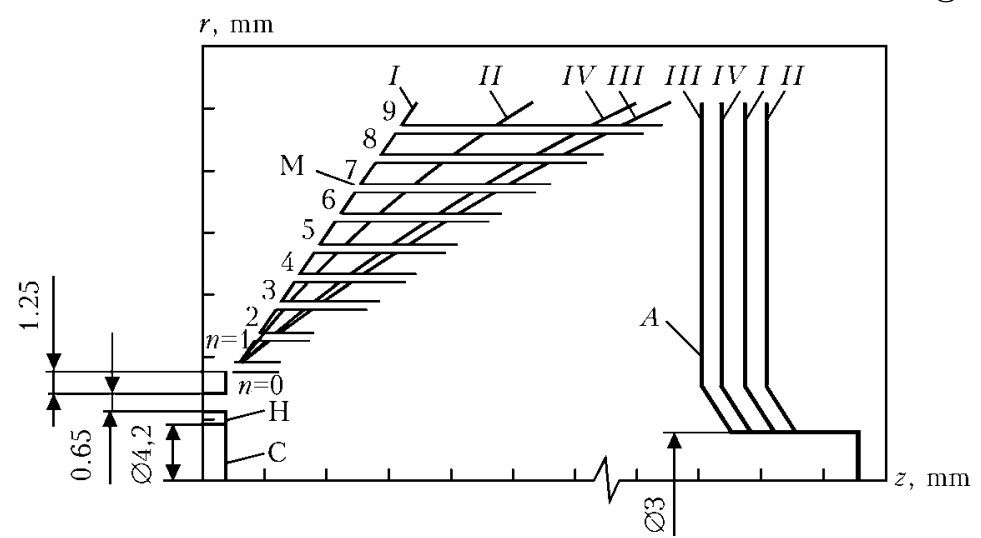

Figure 4. Development of emission system of EBW gun by means of trajectory analysis and synthesis: $H-$ electrode holder; $I-I V-$ successive steps of approximation to desired electrode shape; $n-$ areas for division of focusing electrode contour 


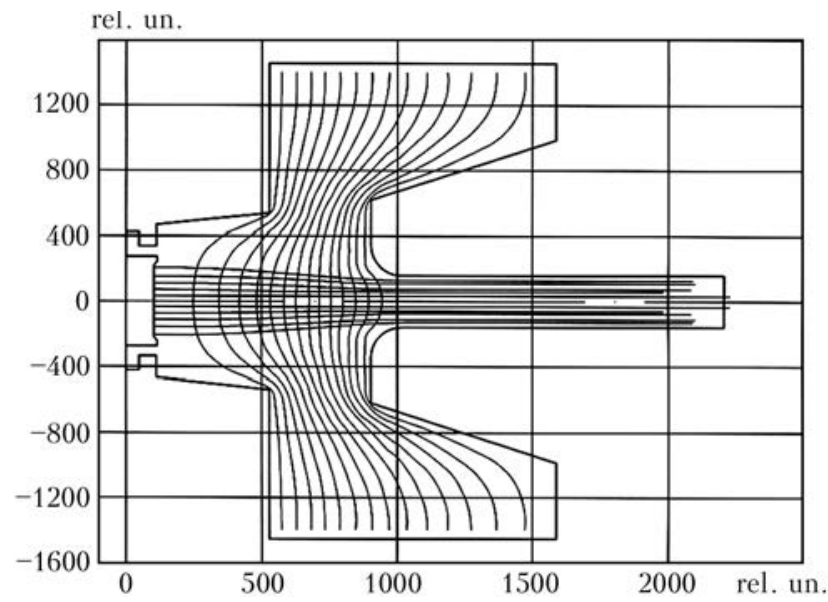

Figure 5. Trajectory analysis of optimized emission system for manual welding gun at $U_{\mathrm{m}}=-100 \mathrm{~V}$ and $I_{\mathrm{b}}=250 \mathrm{~mA}$

perveance at predetermined design-technological limitations on electrode geometry.

The procedure uses a supposition that in calculation focusing electrode can be replaced by composite one, each section of which receives such potentials that their joint effect results in formation of electron beam with set properties.

Required emission system is constructed step by step by means of successive approximation in accordance with algorithm given in the procedure. A sign of finishing convergence processes of the required emission system of construction is close location of resulting contours of focusing electrode and anode after four-five stages of calculation. Thus, for example, in calculation of gun emission system for manual tool the process has converged at stage $I V$ (Figure 4).

Received calculation contour of the focusing electrode is approximated by complex of easy-tomanufacture surfaces (cones, chamfering etc.), and peripheral part of the focusing electrode is taken away from anode in order to increase electric strength of accelerating gap. At the end trajectory analysis of approximated emission system

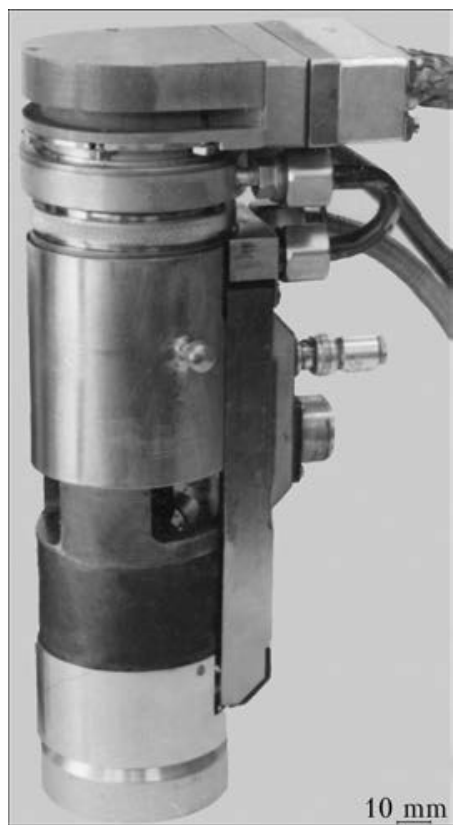

Figure 7. EBW gun used for experimental check-up of optimized emission system

is carried out (beam current $I_{\mathrm{b}}=250 \mathrm{~mA}$ is obtained at cut-off voltage $U_{\mathrm{m}}=-100 \mathrm{~V}\left(\mathrm{Fi}^{-}\right.$ gure 5)). Phase parallelogram is plotted for determination of final parameters of this system (Figure 6).

Thus, for example, length of segment $r$, intercepted by parallelogram sloping sides, is the diameter of beam in crossover $2 r^{*}=0.6 \mathrm{~mm}$. Inclination of median line of the parallelogram helps to evaluate crossover position on system axis relatively to cathode:

$$
\begin{gathered}
c=\frac{r_{\max }^{\prime}}{r_{\max }}=\frac{-1.3 \cdot 10^{-2}}{1.1}=-1.18 \cdot 10^{-2}\left[\frac{1}{\mathrm{~mm}}\right] ; \\
l_{c}=\frac{1}{c}=-\frac{1}{1.18 \cdot 10^{-2}}=-85[\mathrm{~mm}] .
\end{gathered}
$$

Taking into account that phase parallelogram was plotted for section with coordinate $z_{\text {ph.ch }}=$

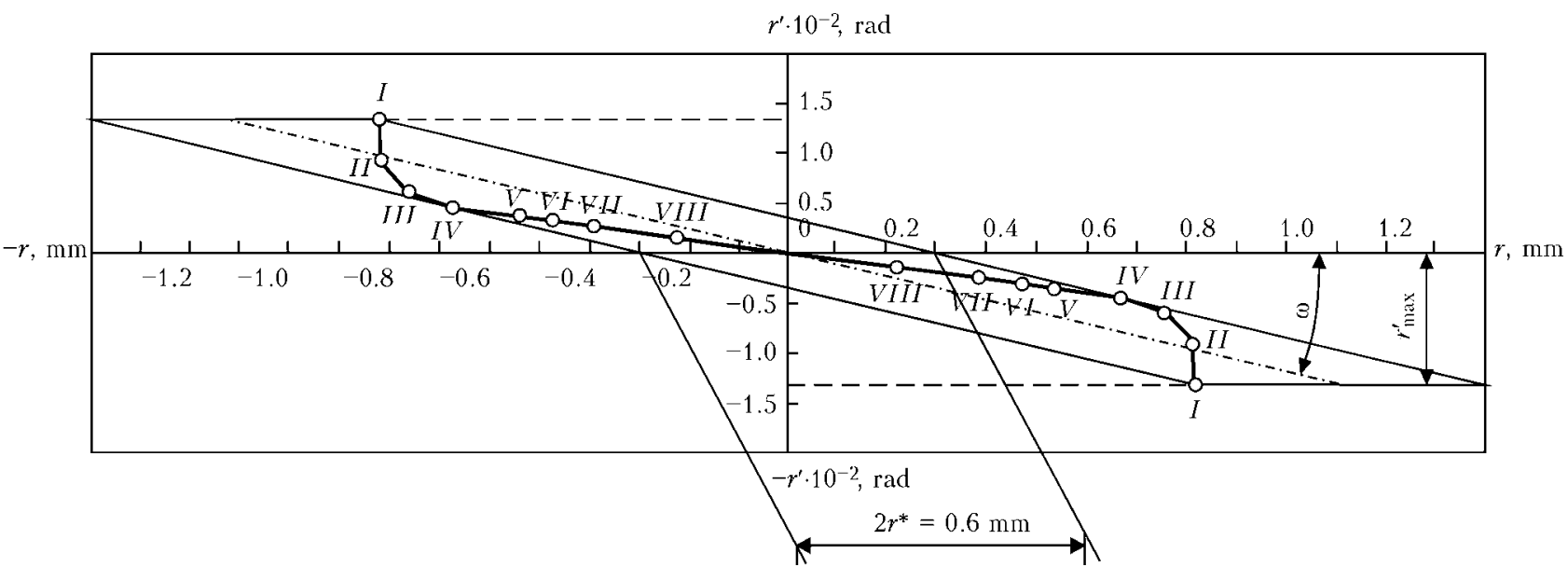

Figure 6. Phase parallelogram plotted on results of trajectory analysis of optimized emission system for longitudinal coordinate $z_{\text {ph.ch }}=19 \mathrm{~mm}$ for manual welding gun 
Parameters of emission systems for manual and automated guns obtained as a results of calculation

\begin{tabular}{|c|c|c|c|c|}
\hline \multirow{2}{*}{ Parameter } & \multicolumn{2}{|c|}{ Manual welding } & \multicolumn{2}{|c|}{ Automated welding } \\
\hline & Set value & Received value & Set value & Received value \\
\hline Perveance, $\mathrm{A} / \mathrm{B}^{3 / 2}$ & $\geq 0.2 \cdot 10^{-6}$ & $0.2 \cdot 10^{-6}$ & $0.2 \cdot 10^{-6}$ & $0.25 \cdot 10^{-6}$ \\
\hline Power beam, kW & $\geq 2.0$ & 2.5 & $\geq 2.0$ & 2.5 \\
\hline Power density in crossover, $\mathrm{kW} / \mathrm{mm}^{2}$ & $\geq 6.0$ & 7.1 & $\geq 7.5$ & 8.8 \\
\hline Half-angle of beam convergence in crossover, rad & $\leq 2 \cdot 10^{-2}$ & $1.32 \cdot 10^{-2}$ & $\geq 10^{-1}$ & $10.3 \cdot 10^{-2}$ \\
\hline Anode-crossover distance, $\mathrm{mm}$ & $\geq 70$ & 85 & $\leq 15$ & 12.7 \\
\hline
\end{tabular}

$=19 \mathrm{~mm}$ (distance from cathode on axis $z$, coordinate $z_{\mathrm{CR}}$ (from cathode) is received: $z_{\mathrm{CR}}=$ $=z_{\text {ph.ch }}-1 / c=19+85=104 \mathrm{~mm}$.

Considering assumed in calculation position of anode inlet $z_{\mathrm{A}}=9 \mathrm{~mm}$ and anode height $\sim 10 \mathrm{~mm}$, gun operating distance $l_{\mathrm{op}}=85 \mathrm{~mm}$ (from anode beam outlet to place of welding) is received. Half-angle of beam convergence, evaluated by extreme trajectory, equals $\sim 1.3 \cdot 10^{-2} \mathrm{rad}$.

The following design-process parameters were set for both variants in development of the emission systems for manual and automated variants of guns (see Figure 4):

- plate cathode of $4.2 \mathrm{~mm}$ diameter;

- ring surface in cathode holder of $0.05^{-}$ $0.1 \mathrm{~mm}$ width;

- annular gap between cathode holder and wall of hole in focusing electrode of $0.65 \mathrm{~mm}$ width;

- flat ring surface in the center of focusing electrode of $1.25 \mathrm{~mm}$ width;

- plate anode with flattened edges of $3 \mathrm{~mm}$ diameter central hole.

The Table provides for set and received calculated parameters of the emission systems for manual and automated guns.

Obtained calculation results were experimentally verified on vacuum unit OB1469M using PL101M gun (Figure 7), which was equipped with developed emission systems for manual and automated welding. Matching on beam current (at cut-off voltage $U_{\mathrm{m}}=-100 \mathrm{~V}$ ) at least $10 \%$, on beam convergence angle at least 5-7\%, on position of beam focal plane at least $15 \%$ were obtained in gun operation (evaluation was made based on penetration of $1 \mathrm{~mm}$ thin inclined plates from stainless steel). Penetration depth of specimens from titanium alloy VT1-0 and aluminum alloy 1201 at $75 \mathrm{~mm}$ working distance in manual variant of gun assembly (without focusing lens) achieved $5 \mathrm{~mm}$ at $2 \mathrm{~kW}$ power in beam and welding speed $25 \mathrm{~m} / \mathrm{h}$. Penetration depth achieved
$6 \mathrm{~mm}$ using additional electromagnetic focusing lens and emission system for automated welding and other conditions being equal.

Thus, the emission systems, developed by methods of trajectory analysis and synthesis, form electron beams sufficient for EBW of metals of up to $4-5 \mathrm{~mm}$ thickness and can be applied in electron gun of a new design for manual and automated welding in space.

Authors express thanks to Prof. V.M. Nesterenkov, Corr. Member of the NASU, Dr. V.E. Lokshin and Eng. V.A. Matvejchuk for useful critical remarks on paper material.

1. Ternovoj, E.G., Shulym, V.F., Lankin, Yu.N. (2013) Repair of fragments of International Space Station using electron beam welding. In: Abstr. of Int. Conf. on Welding and Related Technologies: Present and Future. Kiev: PWI.

2. Paton, B.E., Kubasov, V.N. (1970) Experiment on welding of metals in space. Avtomatich. Svarka, 5, $7-12$.

3. Paton, B.E., Nazarenko, O.K., Chalov, V.I. et al. (1971) Specifics of equipment and technologies of electron beam welding and cutting under space conditions. Ibid., 3, 3-8.

4. (2000) Space: Technologies, materials science, structures: Coll. Ed. by B.E. Paton. Kiev: PWI.

5. Bondarev, A.A., Ternovoj, E.G. (2010) Features of weld formation and properties of aluminium and magnesium alloy joints under simulated space conditions. The Paton Welding J., 11, 16-20.

6. Langmuir, D.B. (1973) Theoretical limitations of cathode-ray tubes. In: Proc. of IRE, 25, 977-991.

7. Gorbenko, N.I., Iliin, V.P., Popova, G.S et al. (1979) Program package ERA for automation of electron-optical calculations. In: Numerical methods of solution of electron optics problems, 31-60. Novosibirsk.

8. Molokovsky, S.I., Sushkov, A.D. (1972) Intensive and ion beams. Leningrad: Energiya.

9. Molokovsky, S.I., Akopiants, K.S., Zubchenko, Yu.V. et al. (1979) Effect of nonlinearity of phase characteristic on focusing of electron beam in welding guns. Avtomatich. Svarka, 9, 14-17.

10. Akopiants, K.S., Zubchenko, Yu.V. (1979) Method of calculation of electron-optical system of welding gun. Ibid., 11, 33-36. 